\title{
Duality for Nonlinear Abstract Evolution Differential Equations
}

\author{
A. Nowakowski and A. RogowskI
}

Es werdèn Existeñz periodischer Lösungen und Dülitätssätze für eine abstrakte Differentialgleichung in einem Hilbért-Raum hergeleitet. Das basiert auf der dualen Methode der Variations: rechnung und einer Modifikation der Tolandschen Dualitätstheorie.

Докавана существование периодических рещении и предложения двонственности для одного абстрактного дифференциального уравнения в гильбертовом пространстве. Для әтого использованы двойственный метод вариационного исчисления и одна модификация теории двоћственности Толанда.

The existence of periodic solutions and duality results for an abstract differential equation in Hilbert space are established. The dual variational method and a modification of the duality theory of Toland are used.

\section{Introduction and statement of the main results}

We shall be dealing with the following $T$-periodic abstract problem in a real separable Hilbert space $X$ :

$$
\begin{aligned}
& d \partial \psi\left(t, x^{\prime}(t)\right) / d t+\partial \psi(t, x(t)) \ni 0, \\
& x(t+T)=x(t)
\end{aligned}
$$

for almost all $t$ in $\mathbb{R}$, where $T$ is a given positive number, $\partial \psi$ and $\partial \vec{\varphi}$ are the subdifferentials of convex lower semicontinuous functions $\varphi(t, \cdot), \varphi(t, \cdot) ; \psi, \varphi: \mathbb{R} \times \mathbb{X} \rightarrow \mathbb{R}$ are $T$-periodic in $t$ and $\mathbb{L} \times \mathbb{B}$-measurable, i.e. measurable with respect to the $\sigma$-algebra generated in $\mathbb{R} \times \bar{X}$ by products of Lebesgue sets in $\mathbb{R}$ and Borel sets in $X$.

To obtain some results for (1.1), we shall consider two functionals: primal

$$
J(x(\cdot))=\int_{0}^{T}\left(-\varphi(t, x(t))+\varphi\left(t, x^{\prime}(t)\right)\right) d t
$$

and dual to it

$$
\begin{aligned}
& J_{\mathrm{D}}(p(\cdot))=\int_{0}^{T}\left(\varphi^{*}\left(t,-p^{\prime}(t)\right)-\psi^{*}(t, p(t))\right) d t+l(p(0), p(T)), \\
& l(a, b)=l_{1}(a)+l_{\mathrm{Q}}(b), \\
& l_{1}(a)=\left\{\begin{array}{ll}
0 & \text { if } a=0, \\
+\infty & \text { if } a \neq 0,
\end{array} \quad l_{2}(b)= \begin{cases}0 & \text { if } b=0, \\
+\infty & \text { if } b \neq 0\end{cases} \right.
\end{aligned}
$$

( $\varphi^{*}$ and $\psi^{*}$ are the Fenchel conjugates of $\left.\varphi(t, \cdot), \psi(t, \cdot)\right)$ both defined on the space $A(X)$ of absolutely continuous functions from $[0, T]$ to $X$ whose squares of norms of derivatives are integrable. 
Note that (1.1 a). is the generalized Euler-Lagrange equation for (1.2) and, further, that the integrand in (1.2) is a concave-convex function. The last fact makes (1.2) indefinite, i.e. it is, in general, unbounded from below and from above. This is the reason why we use the dual functional (1.3) to study (1.1) which is, under some growth conditions on $\varphi$ and $\varphi$, bounded from below in $A(X)$.

The applying of a modification of the duality theory of Touswd $[11,12]$ (see also AUCHMUTY [1]) allows us to reveal the relationships between (1.1), (1.2) and (1.3).

Since the pioneer works of CLARke and Ekexand $[4,5]$ dual variational methods have been extensively used in the study of differential equations (see survey lectures $[2,8]$ ). Our duality principle (see Theorem 1.1 below) differs from that of $[1,2,4,8,12]$. However, it has been pointed out to us by the referee that our Theorem 3.1 may be obtained as a direct application of Tolands duality formula from [11]; but as to the necessary and sufficient optimality conditions compare [7]. But to prove that $J_{\mathrm{D}}$ attains its minimum in $A(X)$ (note that $J_{D}$ is not weakly lower semicontinuous in $A(X)$ ), we shall consider a functional $I$ (see Theorem 3.3) which corresponds in some sense to the dual least action principle for (1.2) (or (1.3)) devised by Clarke. Indeed, the Hamiltonian associated with the integrand of $J$ is $H(t, x, p)=\varphi(t, x)+\psi^{*}(t, x)$; the dual $\mathbf{H a}$ miltonian is $H^{*}(t, \dot{x}, \dot{p})=\varphi^{*}(t, \dot{p})+\varphi(t, \dot{x})$ and it is the main part of the functional $I$. That explains the remark of BREzis [2, p. 415 (bottom)] on the relations between the idea of Toland and that of Clarke and Ekeland.

Let $|\cdot|$ denote the norm of $X$ and $\langle\cdot, \cdot\rangle$ the scalar product in $X$. A measurable function $u: \mathbb{R} \rightarrow X$ will be called $T$-periodic if $u(t+T)=u(t)$ for almost all $t$ in $\mathbb{R}$. The set of all such functions will be denoted by $P(X)$, and $A^{T}(X)$ stands for the subspace of $P(X)$ consisting of all functions which are absolutely continuous in $(i T,(i+1) T)$, $i \in \mathbb{Z}$, and whose squares of norms of derivatives are integrable.

Definition: We say that $x \in A^{T}(X)$ is a solution to (1.1) when there exists some $p \in A^{T}(X)$ such that

$$
\begin{aligned}
& -d p(t) / d t \in \dot{\partial} \varphi(t, x(t)) \text { a.e., } \\
& p(t) \in \partial \psi\left(t, x^{\prime}(t)\right) \quad \text { a.e. }
\end{aligned}
$$

. Now, we state the main result of the paper.

Theorem 1.1: Assume that the following hypotheses are satisfied:

(a) $\dot{\varphi}, \psi$ satisfy the growth conditions.

$$
\because \quad \ldots \varphi(t, x) \leqq(4 l)^{-1}|x|^{2}-d(t), \quad \psi(t, z) \geqq(4 k)^{-1}|z|^{2}-e(t)
$$

for $(t, x),(t, z) \in \mathbb{R} \times \underset{T}{X} ; d, e: \mathbb{R} \rightarrow \mathbb{R}$ are $T$-periodic and summable in $[0, T] ; k>0$ and $\pi^{2} l>k T^{2} ; v \rightarrow \int_{0}^{T} \varphi^{*}(t, v(t)) d t$ is finite on $L^{\infty}(0, T ; B)$, where $B=\{v \in X:|v| \leqq j\}$ for some $j>0$; there exists $z \in L^{2}(0, T ; X)$ such that $\int_{0}^{T} \psi(t, z(t)) d t<\infty$.

(b) $\varphi, \psi$ satisfy the representation

$$
\psi(t, z)=\psi_{1}(t, z)+2^{-1}|z|^{2} \text { and } \varphi^{*}(t, v)=\varphi_{1}(t, v)+a|v|^{2}
$$

$a \geqq T^{2} / 2 \pi^{2}$, where $z \rightarrow \int_{0}^{T} \psi_{1}(t, z(t)) d t$ and $v \rightarrow \int_{0}^{T} \varphi_{1}(t, v(t)) d t$ are sequentially weakly lower semicontinuous in $L^{2}(0, T ; X)$ :

Then there exists a pair $\left(\bar{x}_{T}, \bar{p}_{T}\right)$, being a solution to $(1.1)$, whase restriction $(\bar{x}, \bar{p})$ to $[0, T]$ satisfies

$$
' J_{\mathrm{D}}(\bar{p})=\inf _{p \in A(X)} J_{\mathrm{D}}(p)=\inf _{x^{\prime} \in L^{\prime}} \sup _{x(0) \in X} J(x)=J(\bar{x}) .
$$


Conversely, for any $\bar{p}_{T} \in A^{T}(X)$ for which there exists $\bar{x}_{T} \in A^{T}(X)$ such that their restriction to $[0, T]$ satisfies (1.6), one can choose $\bar{x}_{T} \in A^{T}(X)$ satssfying, together with $\bar{p}_{T^{\prime} ;} ;(1.5)$ and, consequently, (1.1).

\section{Auxiliary results}

Let $L^{2}(0, T ; X)$ and $L^{\infty}(0, T ; X)$ denote the usual Banach spaces! It is known [3] that, for each $x \in A(X)$, the derivative $x^{\prime}(t)$ exists almost every where in $[0, T]$ Therefore $A(X)$ can be identified with $\dot{X} \oplus L^{2}(\hat{0}, T ; X)$ normed by $\|x\|_{A(X)}=\mid x(\hat{0}) \|$ $+\left\|x^{\prime}\right\|_{L^{2}}$. Here $A_{0}(X)$ denotes the subset of $A(X)$ of those $x$ for which $x(0)=x(T)$ $=0$. Let $B^{2}(X)$ be the linear space $X \oplus L^{2}(0, T ; X)$ with the norm $\|c, v\|_{2}=$ max $\left\{|c|,\|v\|_{L^{\prime}}\right\}$. The dual $A^{*}(X)$ of $A(X)$ will be identified with $B^{2}(X)$ under the pairing

$$
\therefore ; \cdots,\langle x ;(c ; v)\rangle=\langle x(0) ; c\rangle+\int_{0}^{T}\left\langle x^{\prime}(t), \dot{v}(t)\right\rangle: d t \text {. }
$$

The conjugate of a function $g: X \rightarrow[-\infty,+\infty]$ is the function $g^{*}: X: \rightarrow[-\infty$, $+\infty]$ defined by $g^{*}\left(x^{*}\right)=\sup \left\{\left\langle x^{*}, x\right\rangle-g(x): x \in X\right\}$; it is lower semicontinuous and convex.

For a concave-convex : function $G: X ; \times \backslash X \rightarrow \mathbb{R}, \partial G(z ; p)$ is the set of all $(u, v), \epsilon$ $\bar{X} \times \bar{X}$ such that

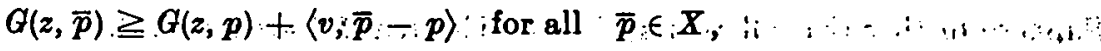

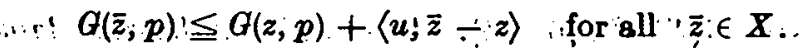

Theorem 2.1 [9]: Let $g:[0, T] \times X \rightarrow \mathbb{R}$ be $\mathbb{L} \times \mathbb{B}-$ measurable and let $g(t, j)$, $t \in[0, T]$, be lower semicontinuous and convex. Assume that $x \rightarrow . \int_{0}^{T} g(t, x(t)) d t$ finite on $L^{\infty}(0, \stackrel{T}{T} ; B), \ddot{B}=\left\{x \epsilon^{\prime} X:|x| \leqq j\right\}$ for a certain $j>0$. Then $t \rightarrow \sup \{g(i, x):|x|$ $\leqq j\}$ is summable in $[0, T]$.

Theorem 2.2 [10]: Letg be the same as in,Theorem 2.1 and assume that there is a $p \in L^{2}(0, T ; X)$ such that $\int_{0}^{T} g^{*}(t, p(t)) d t<+\infty$. Then the functionals $v \rightarrow \int_{0}^{T} g(t, v(t)) d t$ and $p \rightarrow \int_{0} g^{*}(t, p(t)) d t$ are convex and lower semicontinuous in $L^{2}(0, T ; X)$ and they are in duality with respect to the pairing $\langle v, p\rangle=\int_{0}^{r}\langle v(t) ; p(t)\rangle d t$

Lemma 2.1: If $x \in A_{0}(X)$, then i : :

$$
\int_{0}^{T}|x(t)|_{\mid}^{2} d t \leqq\left(T^{2} / \pi^{2}\right) \cdot \int_{0}^{T}\left|x^{\prime}(t)\right|^{2} d t
$$

Proof: Let $x_{k}=(1 / T) \int_{0}^{T} x^{\prime}(t) \exp (-2 k \pi \mathrm{i} t / T) d t, k \in \mathbb{Z}$.

Then expanding $x^{\prime} \in \bar{L}^{2}(0, T ; X)$ in a Fourier series, we get

$$
x^{\prime}(t)=\sum_{k \in \mathbb{Z}} x_{k} \exp (2 k \pi \mathrm{i} t / T), \quad t \in[0, T] .
$$

Since $x \in A_{0}(X)$; therefore $\int_{0}^{T} x^{\prime}(t) d t=0$; so $x_{0}=0$. By integrating (2.3) termwise, 


$$
\begin{aligned}
& x(t)=\sum_{0 \neq k \in \dot{Z}}(T / 2 k \pi \mathrm{i}) x_{k}(\exp (2 k \pi \mathrm{i} / / T)-1) . \text { Hence } \\
& \|x\|_{L^{2}}^{2}=\int_{0}^{T}|x(t)|^{2} d t=\sum_{0 \neq k \in \mathbb{E}}(T / 2 k \pi)^{2}\left|x_{k}\right|^{2}+\left|\sum_{0+k \in \mathbb{E}}(T / 2 k \pi) x_{k}\right|^{2} \\
& \leqq\left(T^{2} / \pi^{2}\right) \sum_{k \in \mathbb{2}}\left|x_{k}\right|^{2}=\left(T^{2} / \pi^{2}\right)\left\|x^{\prime}\right\|_{L^{2}}^{2},
\end{aligned}
$$

and 80 . we have (2.2)

Lem im a 2.2 : Let $c>0, c T^{2} \leqq \pi^{2}$. Then the function

$$
x \rightarrow Q(x)=\int_{0}^{r}\left(\left|x^{\prime}(t)\right|^{2}-c|x(t)|^{2}\right) d t
$$

is convex and lower semicontinuous in $A_{0}(X)$.

: Proof: Let $x, v \in A_{0}(X)$ and let $\alpha \in[0, T]$. Then, using Lemma 2.1, a direct calculation shows

$$
\begin{aligned}
& (1-\alpha) Q(x)+\alpha Q(v)-Q((1-\alpha) x+\alpha v)=(1-\alpha) \alpha Q(x-v) \\
& \geqq(1-\alpha) \alpha\left(\left(\pi^{2} / T^{2}\right)-c\right)\|x-v\|_{L}^{2} \geqq 0,
\end{aligned}
$$

and thus, the convexity of $Q$. Since each of the summands of $Q$ is continuous in $A_{0}(X)$ thus $Q$ is also lower semicontinuous in $A_{0}(X)$

Suppose further that all assumptions of Theorem 1.1 are satisfied. "

Lemma 2.3: Let $S_{b}=\left\{p \cdot \epsilon A(X): J_{\mathrm{D}}(p) \leqq b\right\}, " b>0$. For sufficiently large $b$, $S_{b}$ are non-empty, and bounded in the supremum norm $\|\cdot\|_{c}$. Moreover, $J_{\mathrm{D}}$ is bounded from below.

Proof: Fix $b>, 0$ and take any $p \in A(X)$ such that $J_{D}(p) \leqq b$ (such $b$ and $p$ exist by the assumptions on $\varphi$ and $\varphi$ ). Then, of course, $p(0)=p(T)=0$ and, by Theorem $1.1 /(b)$,

$$
b \geqq J_{D}(p) \geqq l \int_{0}^{T}\left|p^{\prime}(t)\right|^{2} d t+\int_{0}^{T} e(t) d t-k \int_{0}^{T}|p(t)|^{2} d t-\int_{0}^{T} d(t) d t
$$

By Lemma, 2.1 and the inequality $|p(t)|^{2} \leqq T \int_{0}^{T}\left|p^{\prime}(t)\right|^{2} d t$ we further obtain

$$
b+\int_{0}^{T}(d(t)-e(t)) d t \geqq\left(l-k T^{2} / \pi^{2}\right) \int_{0}^{T}\left|p^{\prime}(t)\right|^{2} d t \geqq\left(l / T-k T / \pi^{2}\right)|p(t)|^{2}
$$

for all $t$ in $[0, T]$. Hence we infer the assertions of the lemma

Lemma 2.4 : Let

$$
f(t, z(t))=l\left|p^{\prime}(t)\right|^{2}+e(t)+(4 k)^{-1}\left|x^{\prime}(t)\right|^{2}-d(t)-E\left|x^{\prime}(t)\right| ;
$$

where $z(t)=\left(x^{\prime}(t), p^{\prime}(t)\right)$ and $E>0$. Then the sets

$$
Z_{c}=\left\{z \in L^{2}(0, T ; X \times X): \int_{0}^{T} f(t, z(t)) d t \leqq c\right\}, \quad c \in \mathbb{R},
$$

are either empty or relatively sequentially weakly compact in $L^{2}(0, T ; X \times X)$. Proof: Since $L^{2}(0, T ; X \times X)$ is reflexive, it suffices to note that $\int_{0}^{T} f(t, z(t)) d t /\|z\|_{L^{*}}$
$\rightarrow+\infty$ as $\|z\|_{L^{\prime}} \rightarrow+\infty\left(\|z\|_{L^{2}}=\left\|x^{\prime}\right\|_{L^{\prime}}+\left\|p^{\prime}\right\|_{L^{\prime}}\right)$ 


\section{Duality results}

In what follows we assume that the hypotheses of Theorem 1.1 are satisfied. We define, for each $p \in A(X)$, the perturbation of $J_{\mathrm{D}}$ as

$$
\begin{aligned}
J_{\mathrm{D} p}(a, g)= & -l_{1}(p(0)+a)-l_{2}(p(T)) \\
& +\int_{0}^{T}\left(-\varphi^{*}\left(t,-p^{\prime}(t)\right)+\varphi^{*}(t, p(t)+g(t))\right) d t
\end{aligned}
$$

for $(a, g) \in B^{2}(X)$. Of course, $J_{\mathrm{D}_{p}}(0)=-J_{\mathrm{D}}(p)$. Next, define, for $p, x \in A(X)$,

$$
\begin{aligned}
J_{\mathrm{D} p}^{\#}(x)= & \sup _{g \in L^{2}}\left\{\int_{0}^{T}\left\langle g(t), x^{\prime}(t)\right\rangle d t-\int_{0}^{T}\left(\psi^{*}(t, p(t),+g(t)) \ldots \ldots\right.\right. \\
& \left.\left.-\varphi^{*}\left(t,-p^{\prime}(t)\right)\right) d t+l_{2}(p(T))\right\}+\inf _{a \in X}\left\{\langle a, x(0)\rangle+l_{1}(p(0)+a)\right\} .
\end{aligned}
$$

A direct calculation gives

$$
\begin{aligned}
J_{\mathrm{D} p}^{\#}(x)= & -\langle x(T), p(T)\rangle+l_{2}(p(T)) \\
& +\int_{0}^{T}\left\langle x(t), p^{\prime}(t)\right\rangle d t+\int_{0}^{T} \varphi^{*}\left(t,-p^{\prime}(t)\right) d t+\int_{0}^{T} \psi\left(t, x^{\prime}(t)\right) d t .
\end{aligned}
$$

Thus

$$
\begin{aligned}
\sup _{p \in A(X)}\left\{-J_{D p}^{\#}(x)\right\}= & \sup _{b \in X}\left\{\langle b, x(T)\rangle-l_{2}(b)\right\} \\
& +\sup _{v \in L^{2}}\left\{\int_{0}^{T}\langle v(t), x(t)\rangle d t-\int_{0}^{T} \varphi(t, v(t)) d t\right\}-\int_{0}^{T} \varphi\left(t, x^{\prime}(t)\right) d t \\
& =-\int_{0}^{T}\left(-\varphi(t, x(t))+\varphi\left(t, x^{\prime}(t)\right)\right) d t=-J(x)
\end{aligned}
$$

for $x \in A(X)$. For $p \in A(X)$ and $(a, g) \in B^{2}(X)$, define

$$
\begin{aligned}
J_{D_{p}}^{\# \#}(a, g)= & \sup _{w \in L^{2}}\left\{\int_{0}^{T}\langle g(t), w(t)\rangle d t-\int_{0}^{T}\langle-p(t), w(t)\rangle d t\right. \\
& \left.-\int_{0}^{T} \dot{\psi}(t, w(t)) d t-l_{2}(p(T))-\int_{0}^{T} \varphi^{*}\left(t,-p^{\prime}(t)\right) d t\right\} \\
& +\inf _{x(0) \in X}\{\langle x(0), a\rangle+\langle p(0), x(0)\rangle\}
\end{aligned}
$$

We see that $J_{\mathrm{D} p}^{\# \#}(0)=J_{\mathrm{D} p}(0)$ for all $p \in A(X)$. Using the minimax theorem [3], (3.1) and (3.2), we calculate that

$$
\begin{aligned}
\sup _{p \in A(X)} J_{\mathrm{D} p}^{\# \#}(0) & =\sup _{\boldsymbol{p} \in A(X)} \sup _{x^{\prime} \in L^{\prime}} \inf _{x(0) \in X}\left\{-J_{\mathrm{D} p}^{\#}(x)\right\} \\
& =\sup _{x^{\prime} \in L^{2}} \inf _{x(0) \in X}\{-J(x)\}=-\inf _{x^{\prime} \in L^{2}} \sup _{x(0) \in X} J(x) .
\end{aligned}
$$

A direct consequence of the above considerations is the following

Theorem 3:1: Let $j, J_{\mathrm{L}}$ be as above. Then $\inf _{p \in A(X)} J_{\mathrm{D}}(p) \stackrel{1}{=} \inf _{x^{\prime} \in L^{\prime}} \sup _{x(0) \in X} J(x)$. 
Theorem 3.2: Let $\bar{p} \in A(X)$ be a minimizer for $J_{\mathrm{D}}$ and let $\partial J_{\mathrm{D} \bar{p}}(0)$, be: non-empty. Then there exists $\left(\bar{x}(0), \bar{x}^{\prime}\right) \in \partial J_{\bar{D} \bar{p}}(0)$, where $\bar{x}^{\prime} \in L^{2}(0, T ; X)$ and $\bar{x}(t)=\bar{x}(0)$ $+\int_{0}^{1} \bar{x}^{\prime}(s) d s$, such that $\bar{x} \in A(\bar{X})$ and $J(\bar{x}) \stackrel{1}{=} \inf _{x^{\prime} \in L^{2}} \sup _{x(0) \in X} J(x)$ Furthermore,

$$
J_{\mathrm{D} \bar{p}}(0)+J_{\mathrm{D} \bar{p}}^{\#}(\bar{x})=0, \quad J(\bar{x})-J_{\mathrm{D} \bar{p}}^{\#}(\bar{x})=0 .
$$

Proof: The assumptions on $\varphi^{*}$ and $\psi^{*}$ imply that, for the minimizer $\bar{p}, \bar{p}(0)=\bar{p}(T)$ $=0$. We shall prove that $J_{\mathrm{D}}(\bar{p}) \geqq \sup \{J(\tilde{x}): x(0) \in X\}=J(\bar{x})$, where $\bar{x}(t)=x(0)$ $+\int_{0}^{t} \bar{x}^{\prime}(s) d s$ and $\left.\left(\bar{x}(0), \bar{x}^{\prime}\right)\right)^{\prime} \epsilon j_{\mathrm{D} \bar{p}}(0)$. Put $J_{\mathrm{D}}(\bar{p})=i_{\mathrm{D}}>-\infty$ By the definition of $\partial J_{D p}^{0}$, for the concave-coñuex function $\dot{J}_{\mathrm{D} p}(\cdot, \cdot)$ we have, for each $\left(x(0) ; \bar{x}^{\prime}\right)^{z} \in \partial J_{D \bar{p}}(0)$,

$$
\begin{aligned}
-l_{1}(a) \leqq\langle x(0), a\rangle, \quad \forall a \in X, \quad x(0) \in X, \\
\int_{0}^{T} \psi^{*}(t, \bar{p}(t)+g(t)) d t \geqq \int_{0}^{T} \psi^{*}(t, \bar{p}(t)) d t+\int_{0}^{T}\left\langle g(t), \bar{x}^{\prime}(t)\right\rangle d t
\end{aligned}
$$

for all $g \in L^{2}(0, T ; X)$. The last inequality implies

$$
J_{D \bar{p}}(0, g) \geqq-i_{D}+\int_{0}^{T}\left\langle g(t), \bar{x}^{\prime}(t)\right\rangle d t \quad \forall g \in L^{2}(0, T ; X) .
$$

Further, for $\tilde{x}(t)=x(0)+\int_{0}^{1} \bar{x}^{\prime}(s) d s, x(0) \in X$, we obtain

is...

$$
J_{\mathrm{D} \bar{p}}^{\#}(\bar{x})=\sup _{g \in L^{2}}\left\{\int_{0}^{T}\left\langle g(t), \bar{x}^{\prime}(t)\right\rangle d t-J_{\mathrm{D} \bar{p}}(0, g)\right\} \leqq i_{\mathrm{D}}
$$

Therefore $-J(\tilde{x})=\sup _{p \in A(X)}\left\{-J_{\mathrm{D} p}^{\#}(\tilde{x})\right\} \geqq-i_{\mathrm{D}}$, thus $\sup _{x(0) \in X} J(\tilde{x}) \leqq i_{\mathrm{D}}$

In view of the assumptions on $\varphi$ and the definition of $\varphi^{*}$, we notice that $\varphi(t, \bar{x}(t))$ $+r(t) \geqq j|\tilde{x}(t)|, r(t):=\sup \left\{\varphi^{*}(t, v):|v| \leqq j\right\}$, for $t$ in $[0 ; T]$. The function $r$ is summable in $[0, T]$ (see Theorem 2.1). Observing that $\int_{0}^{T}|\bar{x}(t)| \geqq T\left(|x(0)|-\int_{0}^{T}\left|\bar{x}^{\prime}(t)\right| d t\right)$, we infer that $\int_{0} \varphi(t, \bar{x}(t)) d t \rightarrow+\underset{T}{\rightarrow}$ as $|x(0)| \rightarrow+\infty$. Thus, in virtue of the convexity and lower semicontinuity of $x(0) \rightarrow \int_{0}^{T} \varphi(t, \bar{x}(t)) d t$, we conclude that there exists $\bar{x}(0)$
in $X$ such that

$$
\int_{0}^{T} \varphi(t, \bar{x}(t)) d t=\min _{x(0) \in X} \int_{0}^{T} \varphi(t, \tilde{x}(t)) d t
$$

where

$$
\bar{x}(t)=\bar{x}(0)+\cdot \int_{0}^{t} \bar{x}^{\prime}(s) d s
$$

i.e. $\sup \{J(\bar{x}): x(0) \in \bar{X}\}=J(\bar{x})$. This proves the first assertion of the theorem.

Since $J_{\mathrm{D} \bar{p}}(0)=-i_{\mathrm{D}}$ and $J(\bar{x})=i_{\mathrm{D}}$, therefore $J_{\mathrm{D} \bar{p}}(0)+J(\bar{x})=0$. The inclusion $(\bar{x}(0), \bar{x}) \in \partial J_{D \bar{p}}(0)$ together with the former equality gives (3.4) 
Corollary 3.1: Let $\bar{p} \in A_{0}(X)$ minimize $J_{\mathrm{D}}$ over $A(X)$. Then there exists $\bar{x} \dot{\epsilon} A(X)$, $\bar{x}(t)=\bar{x}(0)+\int_{0}^{t} \bar{x}^{\prime}(s) d s$, such that $\bar{p}(t) \in \partial \psi\left(t, \bar{x}^{\prime}(t)\right)$ and $-\bar{p}^{\prime}(t) \in \partial \varphi(t, \bar{x}(t))$ for almost all $t$ in $[0, T]$, and

$$
J(\bar{x}) \stackrel{=}{=} \inf _{x^{\prime} \in L^{2}} \sup _{x(0) \in X} J(x) \stackrel{\because}{=} J_{\mathrm{D}}(\bar{p}) .
$$

Proof: Since $g \rightarrow \int_{0}^{T} \psi^{*}(t, \bar{p}(t)+g(t)) d t$ is finite in $L^{2}(0, T ; X)$, thus, by [6, Prop. 1.5.2], we see that $\partial \dot{J}_{D \bar{p}}(0)$ is non-empty. Hence and by Theorem 3.2 , there exists $\bar{x} \in A(X)$ satisfying, along .with $\bar{p} \in A(X),(3.4)$ and (3.5). Rewriting (3.4) explicitly, we obtain the inclusions for $\bar{p}$ and $-\bar{p}^{\prime}$, as asserted

Theorem 3.3: Let

$$
\begin{aligned}
I\left(x^{\prime}, p\right)= & l_{1}(p(0))+l_{2}(p(T)) \\
& -\int_{0}^{T}\left\langle p(t), x^{\prime}(t)\right\rangle d t+\int_{0}^{T} \varphi^{*}\left(t,-p^{\prime}(t)\right) d t+\int_{0}^{T} \varphi\left(t, x^{\prime}(t)\right) d t
\end{aligned}
$$

Then

$$
\inf _{\left(x^{\prime}, p\right) \in L^{2} \times A(X)} I\left(x^{\prime}, p\right)=\inf _{p \in A(X)} J_{\mathrm{D}}(p)=\inf _{x^{\prime} \in L^{\circ}} \sup _{x(0) \in X} J(x)
$$

$\left(x(t)=x(0)+\int_{0}^{t} x^{\prime}(s) d s\right)$. Moreover, if $\left(\bar{x}^{\prime}, \bar{p}\right)$ is a minimizer for $I$, then $\bar{p}$ is a, mini mizer for $J_{\mathrm{D}}$.

Proof: By the form of 1 and Theorem 2.2, inf $\left\{I\left(x^{\prime}, p\right): x^{\prime} \in L^{2}\right\}=J_{\mathrm{D}}(p)$ for all $p \in A(X)$, and so we have the first equality. The second one follows from Theorem 3.1.

Suppose that $\left(\bar{x}^{\prime}, \bar{p}\right)$ minimizes $I$ on $L^{2}(0, T ; X) \times A(X)$. Then $I\left(\bar{x}^{\prime}, \bar{p}\right)=\inf \left\{J_{\mathrm{D}}(p)\right.$ : $p \in A(\bar{X})\}$. By the first part of the proof, $J_{\mathrm{D}}(\bar{p})=\inf \left\{I\left(x^{\prime}, \bar{p}\right): x^{\prime}: \epsilon L^{2}\right\} \leqq I\left(\bar{x}^{\prime}, \bar{p}\right)$. Therefore $J_{\mathrm{D}}(\bar{p})=\inf \left\{J_{\mathrm{D}}(p): p \in A(\bar{X})\right\}$

\section{The proof of Theorem 1.1}

We begin with showing that the $I$ defined in Theorem 3.3 is sequentially weakly lower semicontinuous in $L^{2}(0, T ; X) \times A_{0}(\bar{X})$.

By our assumptions; $\varphi^{*}(t, v)=\varphi_{1}(t, v)+a|\dot{v}|^{2}$ and $\psi(t, u)=\psi_{1}(t, u)+2^{-1}|u|^{2}$, where $p^{\prime} \rightarrow \int_{0}^{T} \varphi_{1}\left(t, p^{\prime}(t)\right) d t$ and $x^{\prime} \rightarrow \int_{0}^{T} \psi_{1}\left(t, x^{\prime}(t)\right) d t$ are sequentially weakly lower semicontinuous in $L^{2}(0, T ; X)$. The function $(p, v) \underset{T}{\rightarrow} 2^{-1}|p-v|^{2}$. is convex and lower semicontinuous in $X \times X$, so $\left(x^{\prime}, p\right) \rightarrow 2^{-1} \int_{0}^{T}\left|p(t)-x^{\prime}(t)\right|^{2} d t$ is sequentially weakly lower semicontinuous in $L^{2}(0, T ; X) \times A_{0}(X)$. In virtue of Lemma 2.2, 


$$
\begin{aligned}
& p \rightarrow a \int_{0}^{T}\left(\left|p^{\prime}(t)\right|^{2}-(2 a)^{-1}|p(t)|^{2}\right) d t \text { is also such a function in } A_{0}(X) \text {. Hence we see that } \\
& I\left(x^{\prime}, p\right)= l_{1}(p(0))+l_{2}(p(T)) \\
&+2^{-1} \int_{0}^{T}\left|p(t)-x^{\prime}(t)\right|^{2} d t+\int_{0}^{T}\left(a\left|p^{\prime}(t)\right|^{2}-2^{-1}|p(t)|^{2}\right) d t \\
& \quad+\int_{0}^{T} \varphi_{1}\left(t, p^{\prime}(t)\right) d t+\int_{0}^{T} \psi_{1}\left(t, x^{\prime}(t)\right) d t
\end{aligned}
$$

is, really, sequentially weakly lower semicontinuous in $L^{2}(0, T ; X) \times A_{0}(X)$.

Let now $E>0$ be sufficiently large: Put $D=\{p \in X:|p| \leqq E\}$ and $h(p, u)$ $=\langle p, u\rangle$ if $(p, u)_{T}^{\prime} \in D \times X, h\left(p,{ }^{\prime} u\right)^{\prime}+\infty$ otherwise. Denote by $I_{h}$ the functional $I$ with the term $\int_{0}^{T} h\left(p(t), x^{\prime}(t)\right) d t$ instead of $\int_{0}^{T}\left\langle x^{\prime}(t), p(t)\right\rangle d t$. In view of Lemma 2.3 and Theorem 3.3, inf $I\left(x^{\prime}, p\right)=\inf I_{h}\left(x^{\prime}, p\right)$ on $L^{2} \times A(X)$. Let us take any $\left(x_{b}^{\prime}, p_{b}\right)$ $\epsilon L^{2}(0, T ; X) \times A_{0}(X)$ and put $b=I\left(x_{b}{ }^{\prime}, p_{b}\right)$. Of course, $I\left(x_{b}{ }^{\prime} ; p_{b}\right) \geqq J_{\mathrm{L}}(p)$ for all $p \in A_{0}(X)$. Next, choose $E>0$ so large that each $p \in S_{b}\left(S_{b}\right.$ defined as in Lemma 2.3) is contained in the interior of $D$; this is possible by Lemma 2.3. We easily check that $I_{h}\left(x^{\prime}, p\right) \geqq \int_{0}^{T} f(t, z(t)) d t$ for all $\left(x^{\prime}, p\right) \in L^{2}(0, T ; X) \times A(X)$, where $f$ is defined in Lemma 2.4. By this lemma, the set

$$
M=\left\{\left(x^{\prime}, p\right) \in L^{2}(0, \dot{T} ; X) \times A(X): I_{h}\left(x^{\prime}, p\right) \leqq b\right\}
$$

is relatively sequentially weakly compact in $L^{2}(0, T ; X) \times A(X)$ (in fact, for all $p$ from this set, $p(0)=p(T)=0$, and then, the weak compactness of $p^{\prime}$ in $L^{2}(0, T ; X)$ implies that of $p$ in $A(X))$. Let $\left\{\left(x_{n}{ }^{\prime}, p_{n}\right)\right\} \subset M$ be a minimizing sequence for $I_{n}$. Then, by the above construction, it is also minimizing for $I$, and $I\left(x_{n}{ }^{\prime}, p_{n}\right)=I_{n}\left(\dot{x}_{n}{ }^{\prime}, p_{n}\right)$, $n \in \mathbb{N}$. Since $I$ is sequentially weakly lower semicontinuous and $\left\{\left(x_{n}^{\prime}, p_{n}\right)\right\}$ is relatively sequentially weakly compact, there exists'a subsequence of $\left\{\left(x_{n}^{\prime}, p_{n}\right)\right\}$ converging to some $\left(\bar{x}^{\prime}, \bar{p}\right)$ in $M$ being a'minimizer for $I$.'Hence and by Theorem $3.3 ; \bar{p}$ is 'a minimizer for $J_{D}$.

Further, from Corollary 3.1 we obtain that there exists $\bar{x} \in A(X)$ such that $\overline{\bar{p}}(t)$ $\epsilon \partial_{\varphi}\left(\left(t, \bar{x}^{\prime}(t)\right)\right.$ and $-\dot{\bar{p}}^{\prime}(t) \epsilon \partial \varphi(t ; \bar{x}(t))$ almost everywhere in $[0, T]$, and (3.5) holds.

In view of the $T$-periodicity of the functions $\grave{\varphi}^{*}(\cdot, x), \varphi^{*}(\cdot, x) ; x \in X$; the functions $\varphi^{*}(\cdot, x), \psi(\cdot, x)$ are $T$-periodic, too. Thus each minimizer $\bar{p}$ of $J_{\mathrm{D}}$ can be identified with some $\bar{p}_{T} \in A^{T}(X)$ restricted to $[0, T]$. This means that if we consider the functional

$$
\int_{i \tau .}^{(i+1) T}\left(p^{*}\left(t,-p^{\prime}(t)\right)-\psi^{*}(t, p(t))\right) d t+l(p(i T), p((i+1) T))
$$

$i \in \mathbb{Z}$, instead of $J_{\mathrm{D}}$, then $\bar{p}_{T}$ restricted to $[i T,(i+1) T]$ is a minimizer for it in the space of absolutely continuous functions $p:[i T,(i+1) T] \rightarrow X$. Therefore the $\bar{x}^{\prime} \in L^{2}(0, T ; X)$ from the assertion of Theorem 3.2 can be identified with some function $\bar{x}_{T}^{\prime \prime}: \in \cdot P(X)$ restricted to $[0, T]$. Define

$$
\bar{x}_{T}(t)=\bar{x}(0)+\int_{i T}^{\ell} \bar{x}_{T}^{\prime}(s) d s \quad(t \in[i T,(i+1) T) ;: i \in \mathbb{Z})
$$

Of course, $\bar{x}_{T} \in A^{T}(X)$. Corollary 3.1 implies that $\bar{x}_{T}, \bar{p}_{T}$ satisfy (1.5). The proof of Theorem 1.1 is completed 


\section{Aniexample of équiation (1:1)}

Let $j: \mathbb{R} \times \dot{X} \rightarrow(-\infty ; \infty)$ be bounded, $\mathbb{L}$-measurable and $T$-periodic with respect to the first variable; continuously differentiable and convex with respect to the second 'variable and such that $j(t, r) \geqq e(t),(t, r)^{\prime} \in[0, T] \times \mathbb{R}, e: \mathbb{R} \rightarrow \mathbb{R}$ is $T$-periodic and summable in $T$. We put $\varphi_{1}(t, z)$

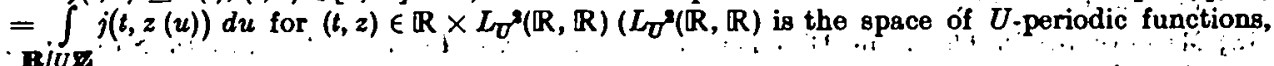
$U>0$, whose restrictions to $[0, \dot{U}]^{\prime}$ belong to $\left.L^{2}(0, U ; \mathbb{R})\right)$. Let $X=H_{U}^{-1}(\mathbb{R}, \mathbb{R})\left(\dot{H} \dot{U}^{\prime}(\mathbb{R} ; \mathbb{R})\right.$ is the Hilbert space of all $U$-periódic functions whose restrictions to $(-M, M)$ belong to the Sóbolev space $H^{1}(-M, M ; \mathbb{R})$ for all $\left.M>0\right)$ with the norm $|z|^{2}=\int_{0}^{U}\left(z_{u}(u)\right)^{2} d u+\int_{0}^{U}(z(u))^{2} d u \psi_{1}(t,)^{\prime}$ is convex and lower semicontinuous in $X ; t, \in[0, T] ; \varphi_{1}$ is also $\mathbb{L} \times \mathbb{B}$-measurable in $\mathbb{R} \times X$. In consequence; $z \rightarrow \int_{0}^{T} \varphi_{1}(t, z(t)) d t$ is sequentially, weakly lower semicontinuous in $L^{2}(0, T ; X)$. We set $\varphi(t, z)=(4 k)^{-1}|z|^{2}+\psi_{1}(t, z), k>0$, for $(t, z) \in \mathbb{R} \times X$. Thus all assumptions of Theorem 1.1 concerning $\varphi$ are satisfied. Now, take $\varphi(t, x)=2^{-1}|x|^{2}$ and assume that $k$ and $T$ are such that $\pi^{2}>2 k T^{2}$ and $\pi \geqq T$. Then we have fulfilled all assumptions of Theorem 1.1. From (1.5a) we calculate for our $\varphi$ that $-p(t)=\int_{0}^{t} x(s) d s$. Therefore by Theorem 1.1 there exists $\bar{x}_{T} \in A^{T}(X)$ whose restriction $\bar{x}$ to $[0, T]$ together with $\bar{p}(t)=-\int_{0}^{t} \bar{x}(s) d s, t \in[0, T]$, satisfy, (1.6) for our $\varphi, \psi$
and it is a solution to the problem

$$
\begin{aligned}
& (d / d t)\left((\partial / \partial r) j\left(t, x_{t}(t, u)\right)\right)+(2 k)^{-1}\left(x_{t}(t, u)-\Delta x_{t}(t, u)\right) \\
& -\Delta x(t, u)+x(t, u)=0, \\
& x(t+T, u)=x(t, u), \quad x(t, u+U)=x(t, u), \quad(t, u) \in \mathbb{R} \times \mathbb{R}, \\
& \int_{0}^{T} x(t, u) d t=0, \quad u \in \mathbb{R},
\end{aligned}
$$

where $\Delta$ is Laplace's operator in $u$.

What is essentially new here is that $\bar{x}$ satisfies (1.6). This is also interesting in its own right as it is not easily to prove directly that the functional

$$
\begin{aligned}
J(x)= & \int_{0}^{T} \int_{0}^{U}\left(j\left(t, x_{t}(t, u)\right)+(4 k)^{-1}\left(\left(x_{t u}(t, u)\right)^{2}+\left(x_{t}(t, u)\right)^{2}\right)\right. \\
& \left.-2^{-1}\left(\left(x_{s}(t, u)\right)^{8}+(x(t, u))^{2}\right)\right) d u d t
\end{aligned}
$$

attains its minimum in any reasonsble space of functions without boundary conditions.

\section{REFERENCES}

[1] Aucemuty, G.: Duality for non-convex variational principles. J. Diff. Equ. 50 (1983), 80 to 145 .

[2] Brezis, H.: Periodic solutions of nonlinear vibrating strings and duality principles. Bull. Amer. Math. Soc. 8 (1983), $409-426$.

[3] Brezts, H.: Operateurs Maximaux Monotones et Semi-Groupes de Contractions dans les Espaces de Hilbert (Math. Studies: Vol. 5). Amsterdam: North-Holland 1973.

[4] Clarke, F. H.: Periodic solution to Hamiltonian inclusion. J. Diff. Equ. 40 (1981), 1-6.

[5] CLARKE, F. H., and I. EK ELAND: Hamiltonian trajectories having prescribed minimal period. Comm. Pure Appl. Math. 33 (1980), 103-116. 
[6] Errmand, I., and R. Tramam: Convex Anslyzis and Variational Problemg: Amsterdam-Oxford: North-Holland Publ. Comp. 1976.

[7] Hranart-Urruty, J.-B.: From convex optimization to nonconvex optimization. Part I: Necessary and sufficient conditions for global optimality. In: Nonsmooth Optimization and Related Topics (Eds.: F. H. Clarke, V. F. Demyanov and F. Giannessi). Now York: Plenum Press 1989.

[8] Mawrin, J.: Problèmes de Dirichlet Variationnels Non Linéaires (Sém. Math. Sup. Univ. Montréal 104): Montréal: Les presses de l’Université 1987.

[9] Nowakowski, A.: Note on convex, integral functionals. Demonstratio Math. 21 (1988), $827-834$.

[10] Rockarkucha, R. T.: Convex: integral ,functionals and duality: In: Contributions to Nonlinear Functional Analysis (Ed.: E. Zarantonello). New York: Academic Press 1971.

[11] Toliand, J: F.: A duality principle for non-convex optimization and the calculus of variations. Arch. Rat. Mech. Anal. 71 (1879), $41-61$.

[12] Tolasd, J. F.: Duality in nonconvex optimization. J: Math. Anal. Appl. 66 (1978), 399 to 415.

Manuskripteingang: 30. 11. 1988; in revidierter Fassung 01. 11.1889

\section{VERFASSER:}

Dr: AxDrzed Nowarrowski und Dr. ANDrzes Rocowski:

Univeraity of Eodé

Institute of Mathematics

ul. S. Banacha 22

PL - 90.238 I $\delta$ d $z$

Poland 